\title{
Human-robot interaction: The impact of robotic aesthetics on anticipated human trust
}

\author{
Joel Pinney ${ }^{\text {Corresp., } 1}$, Fiona Carroll ${ }^{\text {Corresp., } 1}$, Paul Newbury ${ }^{1}$ \\ ${ }^{1}$ Cardiff School of Technologies, Cardiff Metropolitan University, Cardiff, Wales \\ Corresponding Authors: Joel Pinney, Fiona Carroll \\ Email address: st20102131@outlook.cardiffmet.ac.uk, fcarroll@cardiffmet.ac.uk
}

\section{Background}

Human senses have evolved to recognise sensory cues. Beyond our perception, they play an integral role in our emotional processing, learning, and interpretation. They are what help us to sculpt our everyday experiences and can be triggered by aesthetics to form the foundations of our interactions with each other and our surroundings. In terms of Human-Robot Interaction (HRI), robots have the possibility to interact with both people and environments given their senses. They can offer the attributes of human characteristics, which in turn can make the interchange with technology a more appealing and admissible experience. However, for many reasons, people still do not seem to trust and accept robots. Trust is expressed as a person's ability to accept the potential risks associated with participating alongside an entity such as a robot. Whilst trust is an important factor in building relationships with robots, the presence of uncertainties can add an additional dimension to the decision to trust a robot. In order to begin to understand how to build trust with robots and reverse the negative ideology, this paper examines the influences of aesthetic design techniques on the human ability to trust robots.

\section{Method}

This paper explores the potential that robots have unique opportunities to improve their facilities for empathy, emotion, and social awareness beyond their more cognitive functionalities. Through conducting an online questionnaire distributed globally, we explored participants ability and acceptance in trusting the Canbot U03 robot. Participants were presented with a range of visual questions which manipulated the robot's facial screen and asked whether or not they would trust the robot. A selection of questions aimed at putting participants in situations where they were required to establish whether or not to trust a robot's responses based solely on the visual appearance. We accomplished this by manipulating different design elements of the robots facial and chest screens, which influenced the human-robot interaction.

\section{Results}

We found that certain facial aesthetics seem to be more trustworthy than others, such as a cartoon face versus a human face, and that certain visual variables (i.e. blur) afforded uncertainty more than others. Consequentially, this paper reports that participant's uncertainties of the visualisations greatly influenced their willingness to accept and trust the robot. The results of introducing certain anthropomorphic characteristics emphasised the participants embrace of the uncanny valley theory, where pushing the degree of human likeness introduced a thin line between participants accepting robots and not. By understanding what manipulation of design elements created the aesthetic effect that triggered the affective processes, this paper further enriches our knowledge of how we might design for certain emotions, feelings, and ultimately more socially acceptable and trusting robotic experiences.

Peer] Comput. Sci. reviewing PDF | (CS-2021:03:59583:3:0:NEW 29 Nov 2021) 


\section{Human-robot interaction: The impact of robotic} 2 aesthetics on anticipated human trust.

3

4 Joel Pinney $^{1}$, Fiona Carroll ${ }^{1}$, Paul Newbury ${ }^{1}$

5

6

7

8 Corresponding Author:

9 Joel Pinney $^{1}$

10 Western Avenue, Cardiff, CF5 2YB, UK

11 Email address: st20102131@outlook.cardiffmet.ac.uk 


\section{Abstract \\ 44 Background}

45 Human senses have evolved to recognise sensory cues. Beyond our perception, they play an 46 integral role in our emotional processing, learning, and interpretation. They are what help us to 47 sculpt our everyday experiences and can be triggered by aesthetics to form the foundations of 48 our interactions with each other and our surroundings. In terms of Human-Robot Interaction (HRI), 49 robots have the possibility to interact with both people and environments given their senses. They 50 can offer the attributes of human characteristics, which in turn can make the interchange with 51 technology a more appealing and admissible experience. However, for many reasons, people still 52 do not seem to trust and accept robots. Trust is expressed as a person's ability to accept the 53 potential risks associated with participating alongside an entity such as a robot. Whilst trust is an 54 important factor in building relationships with robots, the presence of uncertainties can add an additional dimension to the decision to trust a robot. In order to begin to understand how to build trust with robots and reverse the negative ideology, this paper examines the influences of aesthetic design techniques on the human ability to trust robots.

\section{8}

59

60

61

62

63

64

65

66

67

68

69

70

71

72

73

74

75

76

77

78

79

80

81

82

83

\section{Method}

This paper explores the potential that robots have unique opportunities to improve their facilities for empathy, emotion, and social awareness beyond their more cognitive functionalities. Through conducting an online questionnaire distributed globally, we explored participants ability and acceptance in trusting the Canbot U03 robot. Participants were presented with a range of visual questions which manipulated the robot's facial screen and asked whether or not they would trust the robot. A selection of questions aimed at putting participants in situations where they were required to establish whether or not to trust a robot's responses based solely on the visual appearance. We accomplished this by manipulating different design elements of the robots facial and chest screens, which influenced the human-robot interaction.

\section{Results}

We found that certain facial aesthetics seem to be more trustworthy than others, such as a cartoon face versus a human face, and that certain visual variables (i.e. blur) afforded uncertainty more than others. Consequentially, this paper reports that participant's uncertainties of the visualisations greatly influenced their willingness to accept and trust the robot. The results of introducing certain anthropomorphic characteristics emphasised the participants embrace of the uncanny valley theory, where pushing the degree of human likeness introduced a thin line between participants accepting robots and not. By understanding what manipulation of design elements created the aesthetic effect that triggered the affective processes, this paper further enriches our knowledge of how we might design for certain emotions, feelings, and ultimately more socially acceptable and trusting robotic experiences. 


\section{Introduction}

85 In a world where robotics is becoming more prominent, our ability to trust them has never been 86 so important. With a robot's physical appearance drastically influencing our perceptions of trust, 87 a greater awareness of how design elements and their aesthetic effect may trigger what affective 88

89

90

91

92

93

94

95

96

97

98

99

100

101

102

103

104

105

106

107

108

109

110

111

112

113

114

115

116

117

118

119

120

121

122

123

124

125

126

127 processes are imperative. Robots have an exceptional potential to benefit humans within a team, yet a lack of trust in the robot could result in underutilising or not using the robot at all (Floyd et al., 2014). As Barnes and Jentsch (2010) identified, the key to a successful relationship between man and machines is in how well they can work and adapt to each other. This can develop through the form and structure of the robot that in turn helps establish social expectations. In addition, a robot's morphology can have an effect on its accessibility and desirability (Fong et al., 2003). The research presented in this paper explores how robot aesthetics can heighten participants ability to trust robots. Participants were introduced to an array of robot visualisations (face and chest) and asked to note their impressions towards each visualisation and whether they trusted the robot. This enabled the researchers to investigate how design elements and their combined aesthetic arrangement can act as emotional stimuli and the ability to trust each robot. In detail, by using various design elements (i.e., colour, blurriness, and tone), we were interested in better understanding how we design for the fundamental principles of aesthetic order in human-robotic interaction. We anticipate that uncertainties in and between the visualisations will greatly influence a participant's willingness to accept the robot (i.e., The cohesion of messages, positive and balanced stimuli, non-invasive colours, etc.). This paper highlights not only the impact of risks and uncertainties created by the visualisations on the human-robot interaction but also the potential of robot aesthetics to commence a trusting relationship.

\section{Literature Review}

\section{Human-robot interaction}

Human-Robot Interaction (HRI) is a field dedicated to understanding, designing, and evaluating robotic systems for use by or with humans. (Huang, 2016, p.1). Yanco and Drury (2002) claim that Human-robot interaction is a subset of the field of human-computer interaction $(\mathrm{HCl})$ and that $\mathrm{HRI}$ can be informed by the research in $\mathrm{HCl}$. Scholtz (2002) argues that there are many differences between $\mathrm{HRI}$ and $\mathrm{HCl}$, dependent on dimensions in the environment, system users and physical awareness. 'The fundamental goal of HRI is to develop the principles and algorithms for robot systems that make them capable of direct, safe, and effective interaction with humans.' (Feli-Siefer \& Mataric, 2010, p.86). It is the 'effective interaction' which is of interest to the authors of this paper (i.e., the ability to build a trusting relationship through effective human-robot interaction). HRI quality may be strongly dependent on the capacity of the communication channel(s) to carry information between human and robot (Steinfelf et al., 2016). Robotic communication is based on three components, the channel of communication, communication cues, and the technology that affects transmission. Information can be communicated through three channels: visual, audio, and environmental (Green et al., 2008). The authors of this paper will be focusing on the visual channel of communication and building affective visual communication cues. A socially interactive robot should be able to communicate its trustworthiness through the use of non-verbal signals including facial expressions and bodily

Peer) Comput. Sci. reviewing PDF | (CS-2021:03:59583:3:0:NEW 29 Nov 2021) 
128

129

130

131

132

133

134

135

136

137

138

139

140

141

142

143

144

145

146

147

148

149

150

151

152

153

154

155

156

157

158

159

160

161

162

163

164

165

166

167

168

169

170

171

gestures (Stoeva \& Gelautz, 2020). The face is capable of expressing a range of emotions that others generally have little difficulty identifying (Hancock et al., 2007). Richert et al. (2018) considers these human-like designs combined with the integration of natural user interfaces could enhance the overall acceptance and interaction of these technologies. In more detail, Duffy (2003) states a robot's capacity to be able to engage in meaningful social interaction with people requires a degree of anthropomorphism (human-like qualities). As Gurthrie cited in Daminao and Dumouchel (2018) points out, the tendency to see human faces in ambiguous shapes provides an important advantage to humans, helping them to initially distinguish between friend or enemy and establish an alliance. A robot's appearance at the first interaction can affect how a robot is interpreted by its users, and in turn how the user may interact with the robot (Lupetti, 2017). In terms of human-robot interaction the physical appearance can have an important affect (Canning et al., 2014), yet before humans are able to effectively interact with robots, they must be able to accept and trust them (Billings et al., 2012). This trust is what is of real interest to the authors of this paper, in order to influence how we design for effective trusting relationships between human and robot through their physical and visual appearance.

\section{Aesthetic Interaction}

'Aesthetic interaction is not about conveying meaning and direction through uniform models; it is about triggering imagination, it is thought-provoking and encourages people to think differently about interactive systems, what they do and how they might be used differently to serve differentiated goal' (Petersen et al., 2004, p.271). Aesthetics can be classified as a core principle of design which encompasses a design's visually pleasing qualities, functionality, and emotional considerations (Interaction Design Foundation, n.d.). For many people, an understanding of a robot is achieved through the senses and the reading of bodily form and gestures, facial and chest screens, and sounds as opposed to only the reading of a screen. As a result, it is very important for us to be able to consider the aesthetic processes involved in our interaction with robots. Research shows that aesthetics can afford the construction of associations and meanings through feelings, intuitions, thoughts, memories, etc. (whilst we interact with computers), which we can then stitch together to form a deeper understanding and appreciation of what we are seeing/ experiencing (Carroll, 2010). Indeed, the aesthetic interaction can promote a relationship between the user and the computer (i.e., robot) that encapsulates a person's full relationship - sensory, emotional, and intellectual. In doing so, it can entice an 'engaged interaction' which can change the user's perceptions and interpretations (Carroll, 2010). In our human-robotic interactions, the authors of this paper feel that the aesthetic provides many opportunities to enhance our humanrobotic experiences particularly our trust and acceptability of robots. As Prinz cited in Holmes (2017) points out, our conscious experience consists of perceptions with shades of feelingsobjects (such as robots) can be comforting or scary, sounds are pleasing or annoying, our body feels good or bad - which all can play a crucial role in guiding our behaviours. According to Moors et al. (2013), the basic premise of appraisal theories is that emotions are adaptive responses, which reflect our appraisals of features of the environment/events that are significant for our wellbeing. Essentially, emotions are elicited by evaluations (appraisals) of how events and situations relate to our important goals, values, and concerns. Scherer (2009) suggests that there are four major appraisal objectives that an organism needs to reach to adaptively react to a salient event: relevance (i.e. how relevant is this event for me?), implications (i.e. what are the implications or

Peer) Comput. Sci. reviewing PDF | (CS-2021:03:59583:3:0:NEW 29 Nov 2021) 
172 consequences of this event and how do they affect my well-being, and so on?), coping potential 173 (i.e. how well can I cope with or adjust to these consequences?), and normative significance (i.e. 174 what is the significance of this event for me-concept and for social norms and values?). 175 Interestingly, each emotion has a unique appraisal structure. For example, the aesthetic emotion 176 interest involves two appraisals (Silvia, 2005): appraising an event as new, complex, and 177 unfamiliar (a high novelty-complexity appraisal) and as comprehensible (a high coping-potential 178 appraisal). Interest causes an emotional and motivational state that facilitates exploration, 179 engagement, and learning (Silvia, 2008); it reflects both the emotional and cognitive aspects of 180 engagement (Ainley, 2012). In terms of the aesthetic emotion of knowledge, firstly, the emotions stem from people's appraisals of what they know, what they expect to happen, and what they think they can learn and understand (Silvia, 2009). Secondly, the emotions, for the most part, motivate learning, thinking, and exploring, actions that foster the growth of knowledge (Silvia, 2009). It is generally agreed that the aesthetic information process starts with input from a stimulus, then continues through several processing stages (i.e. Connected to more profound memorial instances) and ends in the final decision-making (i.e. an evaluative judgement of the stimulus) (Markovi'c, 2012). Locher (2015) describes the aesthetic experience as occurring in two stages. Firstly, an initial exposure to the artefact where a viewer spontaneously generates a global impression/gist of the work and secondly, where aesthetic processing ensues (i.e. directed focal exploration to expand knowledge and contribute to a viewer's interpretation, aesthetic judgement, and emotions regarding the artefact). Zajonc (1980), claimed that it is possible for us to like something or be afraid of it before we know precisely what it is and perhaps even without knowing what it is. Since this, there have been many researchers who have begun to explore automatic affective processing; the premise is that beings are able to establish good and bad stimulus before establishing contact with the stimulus (DeHouwer \& Hermans, 2001). In light of this, the evaluation is subject to the interaction between an event and the appraiser (Lazarus,1991). Importantly, the emotions are elicited according to the way a person appraises a situation (Ellsworth, P. C., \& Scherer, 2003). Significantly, however, research shows that certain aesthetic elements can trigger cognitive and affective processes into motion to influence aesthetic appraisals and more especially how a person aesthetically appraises a situation (Blijlevens et al., 2012). In fact, stimuli that evoke aesthetic responses are always composites of multiple elements that do not ordinarily occur together, and when they do, their joint effect is different in kind from the separate effects of the individual elements (Mechner, 2018). In terms of visual elements such as colour, line, form, and composition priming certain emotions, Melcher and Bacci (2013) found that there is a strong bottom-up and objective aspect to the perception of emotion in abstract artworks that may tap into basic visual mechanisms. In his book, James (2018) considered aesthetic emotions to be the immediate and primary sensory pleasure resulting from exposure to a stimulus. Therefore, we ask, can these aesthetic emotions/interactions, in turn, influence how robots are received and how we make decisions to trust them? Indeed, apart from the logical schemes and sense perception, there is also a powerful 'felt' dimension of experience that is prelogical, and that 211 functions importantly in what we think, what we perceive, and how we behave (Cox \& Gendlin, 1963). What is of real importance to the authors of this paper is the interplay between the 214 particular, how the in-take of aesthetic information from a robot's facial and/or chest visualisation 215 can influence how we trust the robot. 
217 Trust, Risk and Uncertainty

218 "Trust is a phenomenon that humans use every day to promote interaction and accept risk in 219 situations where only partial information is available, allowing one person to assume that another 220 will behave as expected." (Cahill et al., 2003, p.53). For many people, trust is the ability to hold a

221

222

223

224

225

226

227

228

229

230

231

232

233

234

235

236

237

238

239

240

241

242

243

244

245

246

247

248

249

250

251

252

253

254

255

256

257

258

259 belief in someone and/or something can be counted upon and dependable, by accepting a level of risk associated with the interaction of another party (Paradeda et al., 2016). A willingness to potentially become vulnerable to the actions of others, based on the expectation that the trusted party will perform actions essential or necessary to the trustor (Mayer et al., 1995). According to Gambetta (2000), trust can be summarised as a particular level of subjective probability with which an agent assesses another in performing a particular action. That trust implicitly means the probability that an action by others will be beneficial enough to consider engaging in cooperation with them despite the risks. Indeed, trust can be evaluated as a probability; however, it is nevertheless a cerebral contract between trustee and trustor that develops within relations between humans (Coeckelbergh, 2012). In terms of the robot aesthetic, the authors of this paper feel that we have a unique opportunity to enrich further our knowledge of how designing for trust may afford a unique robotics experience. In situations such as trusting robots where a person's past behaviours and reputations are unknown, we acquire other sources of information to determine a person's motivations (DeSteno et al., 2012). These other sources of information are used to communicate understanding, which can be done through the use of empathy. As Lee (2006) points out, an agent who appears to be empathetic is perceived as more trustworthy, likeable, and caring. Robots do not possess the ability to build traditional relationships with humans; therefore, they rely heavily on visual appearance to portray their trust. As Lee (2006) reported, human to human perceptions of trust is widely reliant on the empathy they have for one another. Research shows that a common way in which people convey empathy is in the use of their facial expressions (Riek \& Robinson, 2008). In robot-human interaction, research has shown that facial features and expressions can portray important information about others trustworthiness (Valdesolo, 2013). For this paper, it highlights the importance of considering the design elements to initiate positive affective processes. Research by Merritt and Ilgen (2008), shows that widespread implementation of automated technologies has required a greater need for automation and human interaction to work harmoniously together. The conclusion has supported that individuals would use machines more if they are trusted than those they do not. It has generally been agreed that where there is trust, there is a risk; as Gambetta (2000) indicated, trust is a probability; as you determine the level of risk, you can make alternations to the probability of trustworthiness. Lewis et al. (2018) states, the introduction of anthropomorphism poses serious risks, as humans may develop a higher level of trust in a robot than is warranted. Additionally, risks do not always reflect real dangers, but rather culturally framed anxieties originating from social organisation (Wakeham, 2015). Interestingly, research by Robinette et al. (2016) shows that in certain situations, a person may over-trust a robot while mitigating risks and disregarding the prior performance of the robot. However, another dimension of trust is uncertainty. According to Wakeham (2015), who described being uncertain as having an obscured view of the truth, with a limit on what an individual might know. Uncertainty can cause a restriction in the ability to trust; with uncertainty, you are unable to know all that can happen, resulting in trust becoming a leap of faith (Nooteboom, 2019). The decision whether or not to trust a robot based on the uncertainty

Peer) Comput. Sci. reviewing PDF | (CS-2021:03:59583:3:0:NEW 29 Nov 2021) 
260

261

262

263

264

265

266

267

268

269

270

271

272

273

274

275

276

277

278

279

280

281

282

283

284

285

286

287

288

289

290

291

292

293

294

295

296

297

298

299

300

301

302

303

304

presented can trigger ethically adjusted behaviours that aim to avoid dangers and minimise potential risk (Tannert et al., 2007). Viewing uncertainty from a psychological perspective presents both subjective uncertainty and objective uncertainty. Subjective uncertainty represents a person's feelings, while objective uncertainty is concerned with information a person has (Schunn \& Gregory, 2012). In more detail, research has shown how uncertainty influences people's ability to trust (Glaser, 2014), yet in the same way, trust is a way of dealing with uncertainty and objective risks (Frederiksen, 2014).

\section{Materials and Methods}

This study was conducted at Cardiff Metropolitan University from the $31^{\text {st }}$ of March 2020 to the $15^{\text {th }}$ of April 2020 and was designed to capture the perception of participants feelings and attitudes towards trusting robots. The study was conducted using the powerful online survey software: Qualtrics. Participants were selected through stratified random sampling to target both participants with past robotic experience and those without. Through distributing the questionnaire on social media, special robotic interest groups, and online forums, the authors were able to obtain participants from a diverse participant pool. A total of seventy-four participants from the age of 16 plus years (50 Female \& 24 Male) completed the study from a varied demographic. Participants resided globally (i.e., Europe, Africa, Asia, Australia, North America, and South America) and captured an assortment of participants. The questionnaire took approximately thirty minutes in duration. All graphics were generated using Adobe Photoshop, and the study and questions asked had a strong aesthetic visual component.

The study mainly consisted of quantitative questions in order to provide summaries through descriptive statistics. Additionally, an assortment of questions required participants to engage in qualitative questions, which then enabled analysis to enrich interpretations and uncover similarities. The questions were separated into two categories to target both the general acceptance of robotics and specific questions relating to the Canbot U03 robot. In order to not influence a participant's feelings and past experience with robots, the Canbot U03 was not shown during the first block of questions. Participants were provided with a brief definition of trust at the start of the questionnaire "To Believe that someone is good and honest and will not harm you, or that something is safe and reliable" (Cambridge Dictionary, 2021, trust entry).

Once participants had concluded the initial preparatory questions, they were introduced to the opening visual of the Canbot U03 robot. Participants were presented to a Canbot U03 (see Figure 1) with no visual modification and asked whether or not they would trust this robot based on its visual appearance (i.e., only based on the design features). To address the concepts of a participant's ability to trust the Canbot U03 robot, participants were asked to envisage situations in their everyday life where they may encounter a robot. A short list of possible situations and jobs roles were provided to participants (i.e., Teacher, doctor, receptions, bus driver, etc.)."

Peer) Comput. Sci. reviewing PDF | (CS-2021:03:59583:3:0:NEW 29 Nov 2021) 
305

306

307

308

309

310

311

312

313

314

315

316

317

318

319

320

321

322

323

324

325

326

327

328

329

330

331

332

333

334

335

336

337

338

339

340

341

342

343

344

345

346

347

348

349

350

Figure 1

Participants were then presented with a series of questions with different aesthetic modifications throughout. The first modifications came with the Canbot U03 robot presented with a series of cartoon facial expressions portraying different emotions. Participants were prompted to identify the robot's emotion and whether or not they felt the robot was more or less trusting than before. To detail, questions such as the following were asked to participants: How trustworthy is this robot's appearance?, What emotion do you think the robot is feeling?, Does this visual change affect your ability to trust the robot?, How does the robot make you feel with this appearance? Participants were also asked to provide descriptions on the following questions: What characteristics do you believe only robots should have? How do you design a robot that people would trust?

Figure 2:

The following block of questions prompted participants to consider the anthropomorphic characteristics of the robot (see figure 2). Participants were introduced to a series of robots that related to having human features; these questions probed participants for their feelings towards these powerful visual modifications.

The next section of questions was related to how the design element colour influenced the participant's opinions and description of the robot. This required participants to associate words 
351 (i.e., dangerous, happiest, trusting, unpredictable, and unrealistic) with an array of Canbot U03 robots with different colours hues. Participants were presented with eight robot visualisations (see figure 3), all with varying colour hues (i.e., Pink, orange, blue, yellow, etc.) and prompted to associate the expressive wording with an individual Canbot, no Canbot or all Canbots.

Participants were also introduced to a range of visualisations with contrasting images such as conflicting facial expressions and chest screen imagery (i.e., Happy facial expression + Danger symbol on the chest). Participants were asked a series of questions such including: Which Canbot would you describe as most uncertain?, What impact did the cohesion of screens have on your decision? and Does the facial expression overrule the icon on the chest screen when considering the Canbot's emotions? These questions aimed to understand how the level of cohesion between the chest and facial screens can influence a participant's willingness to trust the robot.

Finally, to further probe the concept of risk, participants were presented with mathematical problems that would be too complex for human calculation (i.e., 887x974 \& 997x1066). Participants were then be asked to identify which Canbot $(\mathrm{A}-\mathrm{H})$ displayed the correct solution upon their chest screen. This question required participants to determine the answer they deemed correct based solely on trusting the robot's physical appearance. Optional text boxes were provided throughout the questions to allow participants to expand and express opinions on the robot's appearances.

The Ethics Board at Cardiff Metropolitan University approved the study (CST_2020_Staff_0002), and participants involved were all provided and signed an online consent form to participate in the study and for the academic use of the non-identifiable data.

\section{Results}


395

396

397

398

399

400

401

402

403

404

405

406

407

408

409

410

411

412

413

414

415

416

417

418

419

420

421

422

423

424

425

426

427

428

429

430

431

432

433

434

435

436

437

438
The observations indicate that a participant's willingness to trust a robot was heavily impacted by the aesthetic elements they were exposed to, and whether or not the participant had past experiences with robots. When asked about Figure 1, fifty per cent of participants said they would trust this robot, twenty-eight per cent were unsure, and the remaining twenty-two per cent recorded that they would not trust the robot. Interestingly, anthropomorphism did not encourage more to trust the robot. Figure 2 (Robot B) shows how the introduction of the face impacted participants who first trusted the robot, twenty of the thirty-seven (fifty-four per cent) of participants who first trusted were now non-trusting or uncertain about trusting the robot. However, anthropomorphism did positively influence those unsure of trusting the first robot introduced, with fifty-two per cent changing their opinion from 'unsure' to 'yes' to trust (see figure 4). In the human-like visualisations, it seemed participants had different opinions on how robots should be designed for trust. One participant (P72) said, 'Less human-like as this makes them feel more deceptive' while another described human features as 'creepy' and 'People may become intimidated by implementing human behaviours into a machine'.

\section{Figure 4:}

When probed further into how designing for trust, participants said, 'Give them their own personality that isn't based on human expression 'and that 'human features make the model 'creepy'. One participant notes that the introduction of a realistic human face 'makes people uneasy'. When adding human eyes to the robot visualisation (see figure 2) participants were asked their feelings on the realistic eyes. 80 per cent of participants expressed their dislike of this appearance, making them feel 'confused, scared, worried and surprised'. One participant noted 'the need for distinction between human and robot' and 'the inclusion of human likeness may be intimidating'.

When asked Would you trust this robot? and What do you think this robot feels? 
439

440

441

442

443

444

445

446

447

448

449

450

451

452

453

454

455

456

457

458

459

460

461

462

463

464

465

466

467

468

469

470

471

472

473

474

475

476

477

478

479

480

481

\section{Figure 5:}

In the blurry face visualisations (figure 5), it appeared participants were more apprehensive about trusting the robot. The findings show that half of the participants were able to correctly identify the robot's emotional cue as 'happy' despite the introduction of blurriness. In contrast, the other half of the participants were torn between 'confused, angry, uncertain, uneasy, and uncomfortable' for the robot's emotion. The introduction of the dissimilar stimuli of the happy facial expression and the blurriness presented participants with uncertainties through the contrasting messages each present (i.e., Happy face - trust, blurriness - uncertain). The results were clearer when prompting participants away from identifying which emotion the robot depicted to how these changes made them feel. The participants concerns were expressed when asked about how the Canbot made them feel, with the majority of responses including terms such as uncertain, uneasy, and confused. 
482 In addition, the findings show different impressions towards facial features when faced with the 483 decision to trust (i.e., What robot is providing you with the correct information?). Interestingly sixty484 six per cent of participants selected robot B (Figure 6) as the most trusting, despite the introduction 485 of a hybrid robot (Robot D - Figure 6). Robot five was the next most accepted (fifteen per cent), 486 yet on closer inspection the participant's speed to answer this question was significantly higher 487 (fifty percent increase) than other responses, thus indicating the potential use of a calculator to 488 determine the correct answer to the equation.

489

490

491

492

493

494

495

496

497

498

499

500

501

502

503

504

505

506

507

508

509

510

511

512

513

514

515

516

517

518

519

520

521

522

\section{Figure 7:}

Similar results were seen in Figure 7, with the alterations to the chest screen affording uncertainty to trust the robot to provide the correct answer. We asked participants to indicate which of the six robots posed the correct answer to the equation $997^{*} 1066$ by clicking on the chosen robot. Figure 7 displays the frequency distribution of clicks over the six distinct robot images. Sixty-four per cent of participants selected robot B (the robot with limited visual modifications) as most trusting despite providing incorrect information. Interestingly, robot B presented the incorrect answer to the mathematical equation.

Moreover, participants felt that in order for a robot to be trusting, there is a need for 'a screen that clearly shows the message that is being transmitted' and that 'I would expect the screen display to match with any expressions'. In terms of harmony between face and chest screen, one participant highlighted that 'It would be difficult to trust a robot with a face and another image within the robot screen. I would trust better with just one option.' In particular, when exposed to figure 8, participants felt that the facial expressions produced a contradicting message to the one upon the chest screen. With sixty per cent of participants declaring the robot as untrustworthy and a further thirty-eight per cent unsure whether or not to trust the robot. One participant could not trust the robot as 'I could not take anything this bot says seriously with that expression'. This highlights the true impact of the misaligned messages on participants ability to trust. 
536

\section{Discussion}

538 In this study, we investigated the impact of the aesthetic order of facial and chest visualisations on participants willingness to trust robots. In particular, it considered the potential risks and

540

541

542

543

544

545

546

547

548

549

550

551

552

553

554

555

556

557

558

559

560

561

562

563

564

565

566 uncertainty afforded by certain aesthetic orders to the human-robotic trusting relationship. Our results show the clear influence that past experience had on a participant's willingness to trust the original robot. Particularly, the visualisation with no modifications was found to have a substantially higher percentage of trust in those with past experience. Participants with no past experiences were relying solely on the visual appearance to determine their level of trust. These findings are in line with what Sanders et al. (2017) hypothesised and discovered, in detail, how those participants with past robotics experience would lead to a higher trust of robots and a better positive attitude towards them.

Interestingly, we found that a blurred facial expression significantly influenced whether participants trusted a robot. The blurred facial and chest screen visualisations afforded uncertainty and resulted in a participant's unwillingness to trust a robot.

Figure 7 displayed the extent that physical appearance had on the decision participants made to trust a robot. With a participant's ability to roughly estimate the correct answer not largely adopted by participants, we can only conclude that the physical appearance was the determining factor in the decisions. Interestingly, over half the participants selected robot B, which presented the incorrect answer to the mathematical equation.

Based on previous research that shows colours can influence various moods (Kurt \& Osueke, 2014), we predicted similarly that the aesthetic element colour could initiate different affective responses when applied to a robot. We tested that hypothesis by introducing participants to an array of robot visualisations that applied an assortment of distinct colour changes. We found that comparably participants were following known psychology of colour associations when selecting what feelings and terms they associated to the robots with the assortment of colours. For example, figure 9 displays the words participants associated with the array of colours and other visual modifications. As we hypothesized, certain colours had followed the known associations of related words, such as when participants were promoted to associate the red coloured robot to a particular word. Following the commonly known western culture word associations with the colour 
567

568

569

570

571

572

573

574

575

576

577

578

579

580

581

582

583

584

585

586

587

588

589

590

591

592

593

594

595

596

597

598

599

600

601

602

603

604

605

606

607

608

609

610 red (i.e., dangerous, excitement, festive, etc.) (Cousins, 2012), we evaluated its affect while present on a robots outer shell and found a similar result of red being associated with the term dangerous. 
611 by the facial expression demonstrated that most participants declared the robot as not trustworthy.

612 Moving forward, when designing a robot that can be trusted, it is important to consider all 613 elements, as stimuli from other visual outputs can potentially influence an independent

614 communication channel.

615

616 Conclusion and future work

617

618 This research has shown that robots have the unique ability to create an emotional connection 619 with humans through the use of facial expressions and aesthetics. As documented, we have seen 620 the introduction of anthropomorphism which creates a fine line between increasing 621 trustworthiness and becoming 'scary'. Nevertheless, the non-physical humanlike 622 anthropomorphic designs (cartoon designs) encouraged participants to trust the robots further, 623 showing the unique ability to improve their facilities for empathy. Moreover, this research has 624 shown that the face is not the sole visual aesthetic that can be utilised to initiate affective 625 processes. The chest screen provides an additional entity to further enrich the potential to provide 626 an engaging experience. Ultimately, the cohesion between the multiple screens is an important 627 consideration for designing socially acceptable robots. As is the design elements and principles 628 to understand how their aesthetic order can play such an important role in initiating a trusting 629 robotic experience.

630

631

Going forth, we feel there may be interest in replicating the study but utilising actual robots. We acknowledge there is still a substantial amount of research required to fully understand how we form trusting relationships between human and robot. However, we feel this study paves the way for future studies that involve aesthetic physicalisation, where further sensory cues can be tested to evaluate their influence on our trusting ability of robots. Additionally, this research touched upon how design elements may influence different participants from different cultural backgrounds, geographical locations, and beliefs. We feel it would be of interest to further explore the potential to develop culturally appropriate robots.

639 Moreover, it would be interesting to further expand on the use of aesthetic designs to evaluate 640 how further modifications (i.e., different colour tones, design elements, design principles, etc.) can 641 affect and in some cases, increase a participant's willingness to trust a robot.

642 Finally, we believe there would be value in understanding how the trusting relationship between 643 human and robot may develop over time. Whilst this study provides details on the initial 644 engagement/interaction, there may be interest to explore aesthetic designs in different situations 645 and time scales.

646 Throughout this research, we have explored how we can build trusting relationships with robotics 647 through aesthetic designs. In future work, better consideration of human-centered design 648 perspectives must be explored when considering building trust. The research explores 649 participants not trusting robotics as injudicious when the reason not to trust is still a valid and 650 acceptable response in certain situations.

651

652

\section{Acknowledgements}

653 
654 We would like to take this opportunity to thank the Eureka Robotics Lab for their continued

655 support. As one of the flagship research clusters at the Cardiff School of Technologies,

656 EUREKA Robotics Lab is the innovative research hub nested in the School of Technologies,

657 Cardiff Metropolitan University, serving Wales and global stakeholders (EUREKA Robotics Lab, 658 2021).

659

660

\section{References}

661

662

663

664

665

666

667

668

669

670

1. Floyd, M., Drinkwater, M.W., Aha, D. W. (2014). Case-based behavior adaptation using an inverse trust metric, AAAI Workshop - Technical Report.

2. Barnes, M., Jentsch, F. (2010). Human-Robot Interactions in Future Military Operations, Ashgate Publishing Company.

3. Fong, T., Nourbakhsh, I., Dautenhahn, K. (2003). A survey of socially interactive robots, Robotics and Autonomous Systems,42, 3-4.

4. Huang, W. (2016). When HCI Meets HRI: the intersection and distinction, Proceedings of the 9th Nordic Conference on Human-Computer Interaction, 1-8.

671

5. Yanco, H. A., Drury, J. L. (2002). A Taxonomy for Human-Robot Interaction Engineering.

672

673

674

6. Scholtz, J. (2002). Human Robot Interactions: Creating Synergistic CyberForces, MultiRobot Systems: From Swarms to Intelligent Automata, 177-184.

7. Feli-Siefer, D., Mataric, M.J. (2010). Human-robot interaction, IEEE Robotics and Automation Magazine 17(2).

675

676

677

8. Steinfeld, A., Fong, T., Kaber, D., Lewis, M., Scholtz, J., Schultz, A., Goodrich, M. (2006). Common metrics for human-robot interaction, HRI 2006: Proceedings of the 2006 ACM

678

679

680

681

682

683 Conference on Human-Robot Interaction, 33-40.

9. Green, S. A., Billinghurst, M., Chen, X., Chase, J. G. (2008). Human-Robot Collaboration: A Literature Review and Augmented Reality Approach inDesign, International Journal of Advanced Robotic Systems, 5, 1-18.

684

10. Stoeva, D., Gelautz. M. (2000). Body language in affective human-robot inter-action, ACM/IEEE International Conference on Human-Robot Interaction, 606-608.

11. Hancock, J. T., Landrigan, C., Silver, C. (2007). Expressing emotion in text-based communication, Conference on Human Factors in Computing Systems -Proceedings, 929932. 
686

687

688

689

690

691

692

693

694

695

696

697

698

699

700

701

702

703

704

705

706

707

708

709

710

711

712

713

714

715

716

12. Richert, A., Müller, S., Schrode, S., Jeschke, S. (2018). Anthropomorphism in social robotics: empirical results on human-robot interaction in hy-brid production workplaces, AI, and Society 3 (3), 413-424.

13. Duffy, B. R. (2003). Anthropomorphism and the social robot, Robotics and Autonomous Systems, 42, (3-4), 177-190.

14. Guthrie, L. cited in Damiano, P. Dumouchel. (2018). Anthropomorphism inhuman-robot co-evolution, Frontiers in Psychology 9, 1-9.

15. Luptetti, M. L. (2017). Available at https://interactions.acm.org/blog/view/robotsaesthetics-and-heritage-contexts (accessed 05/05/2020).

16. Canning, C., Donahue, T. J., Scheutz, M. (2014). investigating humanperceptions of robot capabilities in remote human-robot team tasks based on first-person robot video feeds, IEEE International Conference on Intelligent Robots and Systems, 4354-4361.

17. Billings, D. R., Schaefer, K. E., Chen, J. Y., Hancock, P. A. (2012). Human-robot interaction: Developing trust in robots, HRI'12 - Proceedings ofthe 7th Annual ACM/IEEE International Conference on Human-RobotInteraction, 109-110.

18. Cahill, V., Gray, E., Seigneur, J. M., Jensen, C. D., Chen, Y., Shand, B., Dimmock, N., Twigg, A., Bacon, J., English, C., Wagealla, W., Terzis, S., Nixon, P., Serugendo, G. Di Marzo., Bryce, C., Carbone, M., Krukow, K., Nielsen, M. (2003). Using trust for secure collaboration in uncertain environments, IEEE Pervasive Computing 2 (3).

19. Paradeda, R. B., Hashemian, M., Rodrigues, R. A., Paiva, A. (2016). how facial expressions, and small talk may influence trust in a robot, Lecture Notes in Computer Science, 9979 LNAI, 169-178.

20. Mayer, R. C., Davis, J. H, Schoorman, F. D. (1995). Model of Trust, Management 20 (3), 709-734.

21. Gambetta, D. (2000). Can We Trust? Trust: Making and Breaking Co-operative Relations, $213-237$.

22. DeSteno, D., Breazeal, C., Frank, R.H., Pizarro, D., Baumann, J., Dickens, L., Lee, J.J. (2012). Detecting the Trustworthiness of Novel Partners in Economic Exchange, Psychological Science 23 (12), 1549-1556.

23. Lee, B. (2006). Empathy, androids and 'authentic experience', Connection Science 18 (4), 419-428. 
717 24. Riek, L. D., Robinson, P. (2008). Real-time empathy: Facial mimicry on a robot, ACM 718 Workshop on Affective Interaction in Natural Environments (AFFINE) at the International 719 ACM Conference on Multimodal Inter-faces (ICMI 08), 1-5.

720

721

25. Valdesolo, P. (2013). what body language indicates 'Trustworthy', Available at

722 https://www.scientificamerican.com/article/psychologist-uncover-hidden-signals-of-trust-

723 using-a-robot/, (accessed 04/05/2020).

725

26. Merritt, S.M., Ilgen, D.R. (2008). not all trust is created equal: Dispositional and historybased trust in human-automation interactions, Human Fac-tors 50 (2), 194-210.

726

27. Lewis, M., Sycara, K., Walker, P. (2018). The Role of Trust in Human-RobotInteraction,

727

728 Studies in Systems, Decision and Control, 117, 135-159.

28. Wakeham, J. (2015). Uncertainty: History of the Concept, second edi Edition, no. November, Elsevier.

729

29. Robinette, P., Li, W., Allen, R., Howard, A M., Wagner, A. R. (2016). Overtrust of robots in emergency evacuation scenarios, ACM/IEEE International Conference on HumanRobot Interaction, 101-108.

30. Nooteboom, B. (2019). Uncertainty and the Economic Need for Trust, Vol. 42, Brill, BOSTON.

31. Tannert, C., Elvers, H., Jandrig, B. (2007). The ethics of uncertainty, EMBO reports8 (10), 892-896.

32. Glaser, J.E. (2014). Conversational Intelligence: How great leaders build trust \& get extraordinary results.1st edn. Bibliomotion, New York.

33. Frederiksen, M. (2014). Trust in the face of uncertainty: a qualitative study of intersubjective trust and risk, International Review of Sociology 24 (1), 130-144.

741

34. Sanders, T. L., MacArthur, K., Volante, W., Hancock, G., MacGillivray, T., Shugars, W., Hancock, P.A. (2017). Trust, and prior experience in human-robot interaction, Proceedings of the Human Factors and Ergonomics Society, 1809-1813.

35. Ainley, M. (2012). Students' interest and engagement in classroom activities. In Handbook of Research on Student Engagement.

36. Blijlevens, J., Carbon, C. C., Mugge, R., and Schoormans, J. P. (2012). Aesthetic appraisal of product designs: Independent effects of typicality and arousal. British Journal of Psychology. 
748

749

750

751

752

753

754

755

756

757

758

759

760

761

762

763

764

765

766

767

768

769

770

771

772

773

774

775

776

777

778

37. Carroll, F. (2010). Designing (for) experiences in photorealistic VR environ-mesne Review of Hypermedia and Multimedia.4

38. Cox, J. W. R. and Genelin, E. T. (1963). Experiencing and the Creation of Meaning: A Philosophical and Psychological Approach to the Subjective. The Philosophical Quarterly

39. De Houser, J. and Hermans, D. (2001). Automatic affective processing. Cognition and Emotion.

40. Ellsworth, P. C., \& Scherer, K. R. (2003). Appraisal processes in emotion. In Handbook of affective sciences.

41. James, W. (2018). The principles of psychology. Personality. Culture. Society.

42. Lazarus, R. (1991). The Language of Displayed Art. New York: Oxford University Press.

43. Locher, P. J. (2015). The Aesthetic Experience with Visual Art at FirstGlance.

44. Markovi' c, S. (2012). Components of aesthetic experience: Aesthetic fascination, aesthetic appraisal, and aesthetic emotion. I-Perception, 3(1):1-17.

45. Mechner, F. (2018). A Behavioral and Biological Analysis of Aesthetics: Implications for Research and Applications. Psychological Record.

46. Melcher, D. and Bacci, F. (2013). Perception of emotion in abstract artworks: A multidisciplinary approach. InProgress in Brain Research.

47. Moors, A., Ellsworth, P. C., Scherer, K. R., and Frijda, N. H. (2013). Appraisal theories of emotion: State of the art and future development. Emotion Review.

48. Petersen, M. G., Iversen, O. S., Krogh, P. G., and Ludvigsen, M. (2004). Aesthetic interaction - A pragmatist's aesthetics of interactive systems.

49. Prinz cited in Holmes, B. (2017). Why be conscious? . . New Scientist, 234 (3125), 2832.

50. Scherer, K. R. (2009). Emotions are emergent processes: They require adynamic computational architecture.

51. Silvia, P. J. (2005). What is interesting? Exploring the appraisal structure of interest. Emotion.

52. Silvia, P. J. (2008). Appraisal components and emotion traits: Examining the appraisal basis of trait curiosity. Cognition and Emotion.

53. Silvia, P. J. (2009). Looking Past Pleasure: Anger, Confusion, Disgust, Pride, Surprise, and Other Unusual Aesthetic Emotions. Psychology of Aesthetics, Creativity, and the Arts. 
779 780

781

782

783

784

785

786

787

788

789

790

791

792

793

794

54. Zajonc, R. B. (1980). Feeling and thinking: Preferences need no inferences. American Psychologist.

55. C. D. Schunn and T. J. Gregory. (2012). "The psychology of uncertainty in scientific data analysis No Title," in Handbook of the Psychology of Science., F. Feist and M. Gorman, Eds. Springer Publishing, pp. 461-485.

56. Coeckelbergh “Can we trust robots?” Ethics Inf. Technol., vol. 14, no. 1, pp. 53-60, 2012, doi: 10.1007/s10676-011-9279-1.

57. Cousins, C. (2012). Color and Cultural Design Considerations. Available at: https://www.webdesignerdepot.com/2012/06/color-and-cultural-designconsiderations/\#: : text=Western\%20cultures $\% 20$ (North\%20America $\% 20$ and,Eastern $\% 2$ 0block\%2C\%20it\%20represents\%20communism. (Accessed 10/03/2021).

58. Kurt, S., and Osueke, K.K. (2014). "The Effects of Color on the Moods of College Students," SAGE Open, vol. 4, no. 1.

59. Cardiffmet.ac.uk. (2021). EUREKA Robotics Lab. [online] Available at: $<$ https://www.cardiffmet.ac.uk/technologies/Pages/EUREKA-Robotics-Lab.aspx> [Accessed 17 March 2021].

795 
Figure 1

Original image of the Canbot-U03 robot 


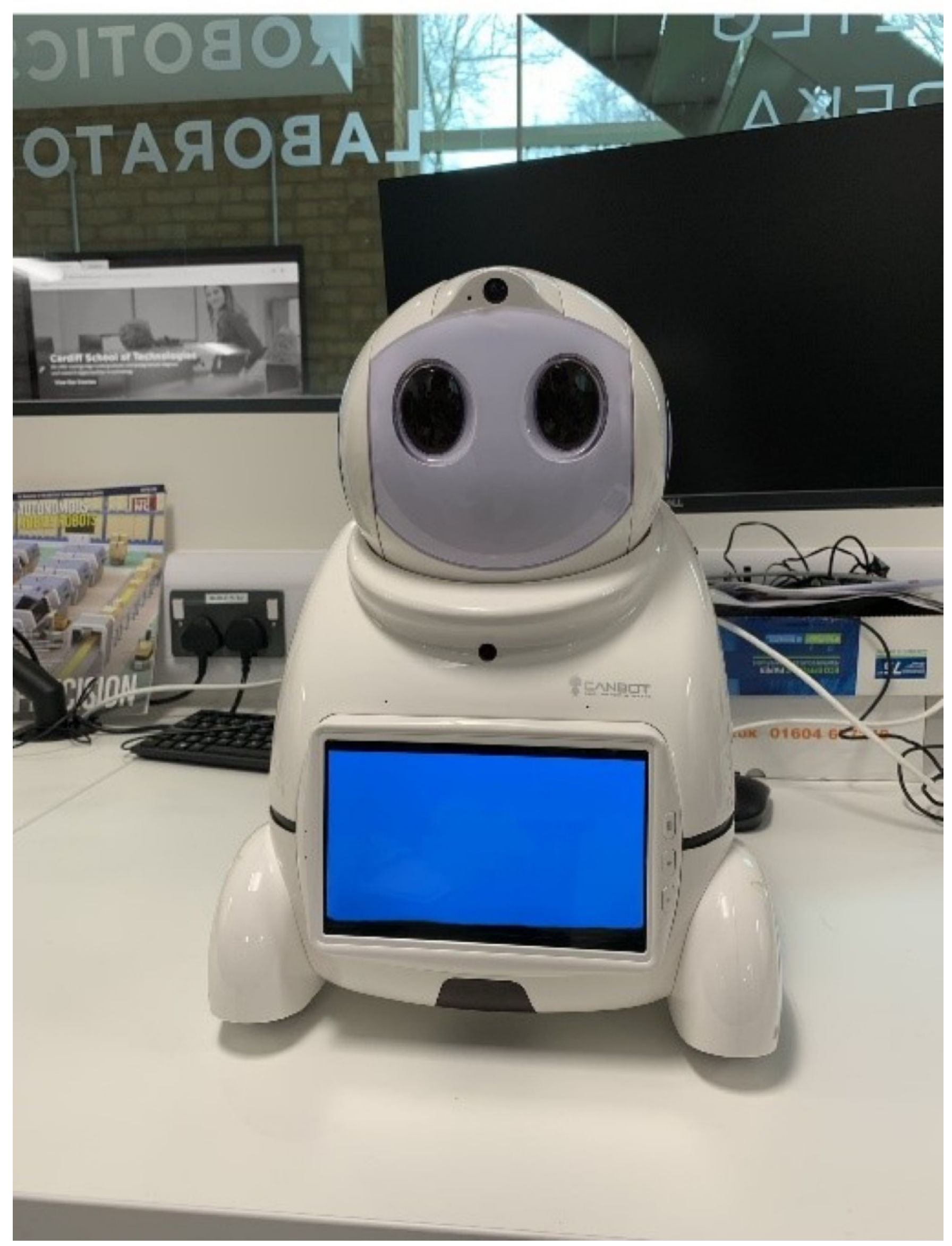


Figure 2

Canbot-U03 robot with human eyes modification. 


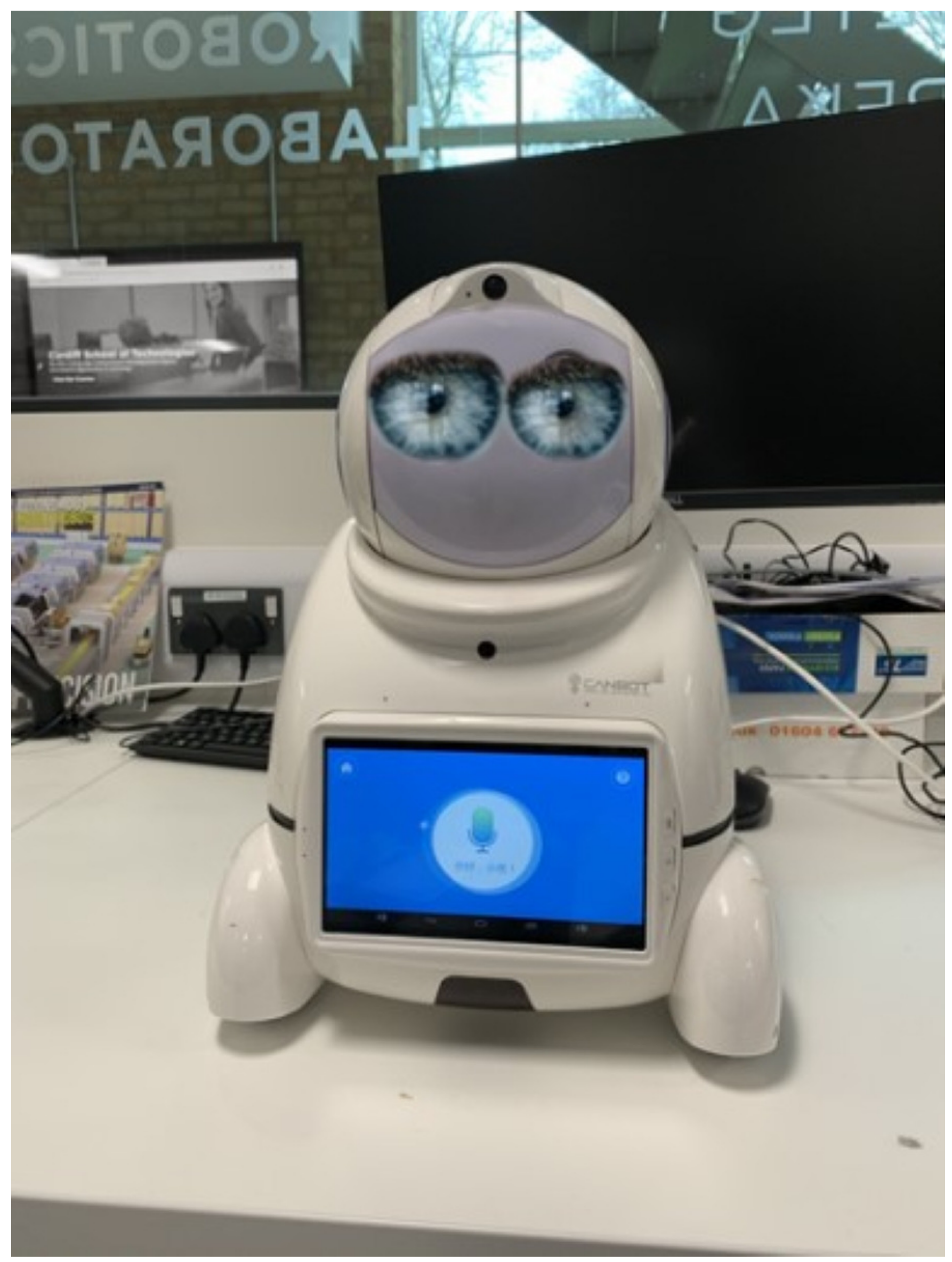


Figure 3

Multiple Canbot-U03 robots with different colours hues.

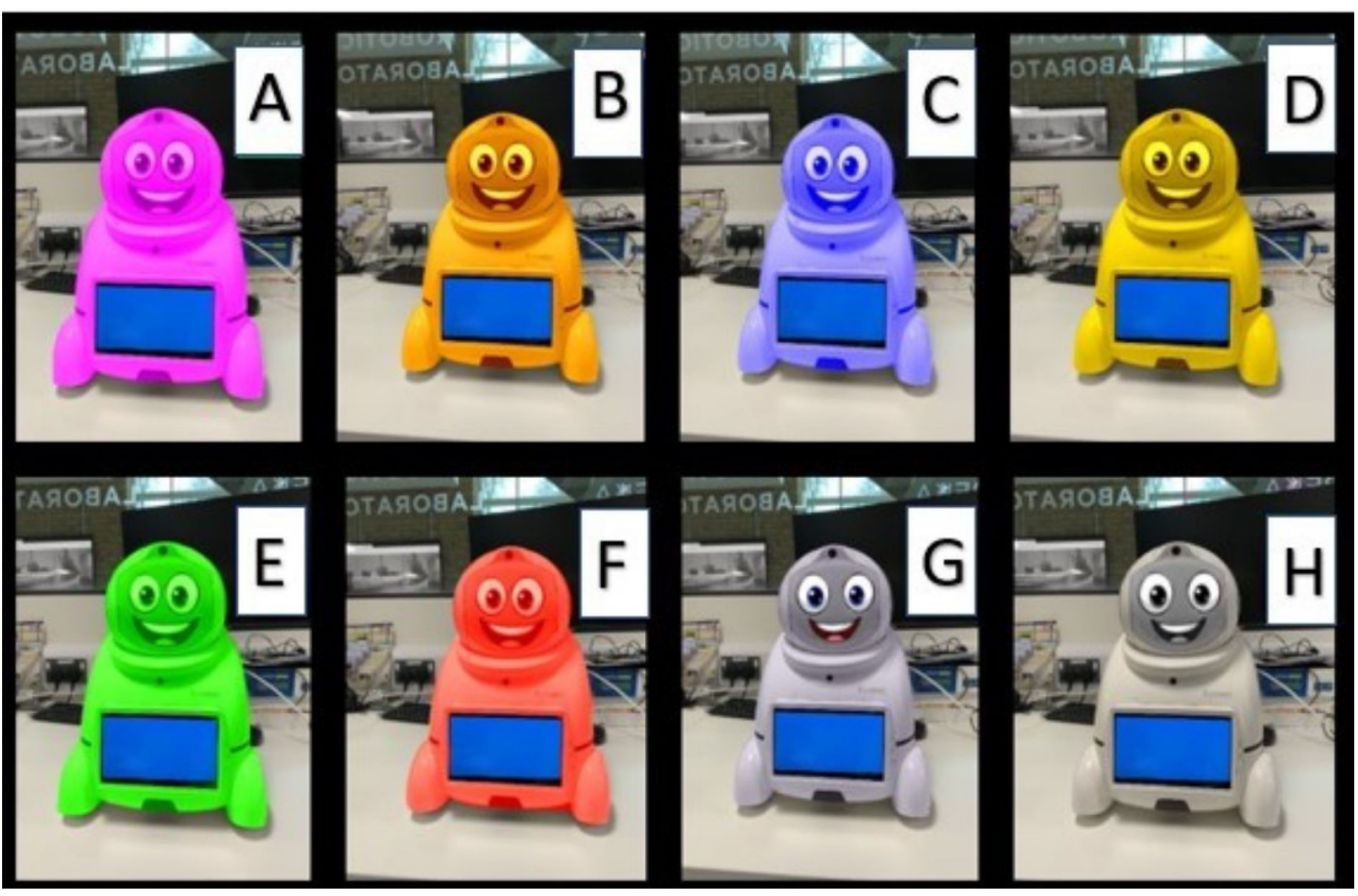


Figure 4

Question to participants: Would you trust this Canbot with the visual changes?

(A) Indicates participants responses to Canbot with no visual changes. (B) Indicates participants responses to Canbot with smiling cartoon facial expression.

\begin{tabular}{|c|c|c|}
\hline & $\begin{array}{c}\text { Robot (A) with no visual } \\
\text { changes (original robot) }\end{array}$ & $\begin{array}{c}\text { Robot (B) with enhanced } \\
\text { visual changes (Cartoon } \\
\text { smiling facial expression) }\end{array}$ \\
$\begin{array}{c}\text { Would you trust this } \\
\text { robot? }\end{array}$ & \\
\hline YES & 37 & 34 \\
\hline No & 16 & 28 \\
\hline Don't Know & 21 & 12 \\
\hline
\end{tabular}




\section{Figure 5}

Canbot with Blurry facial expression and tree map diagram displaying responses from "How does this Canbot make you feel?
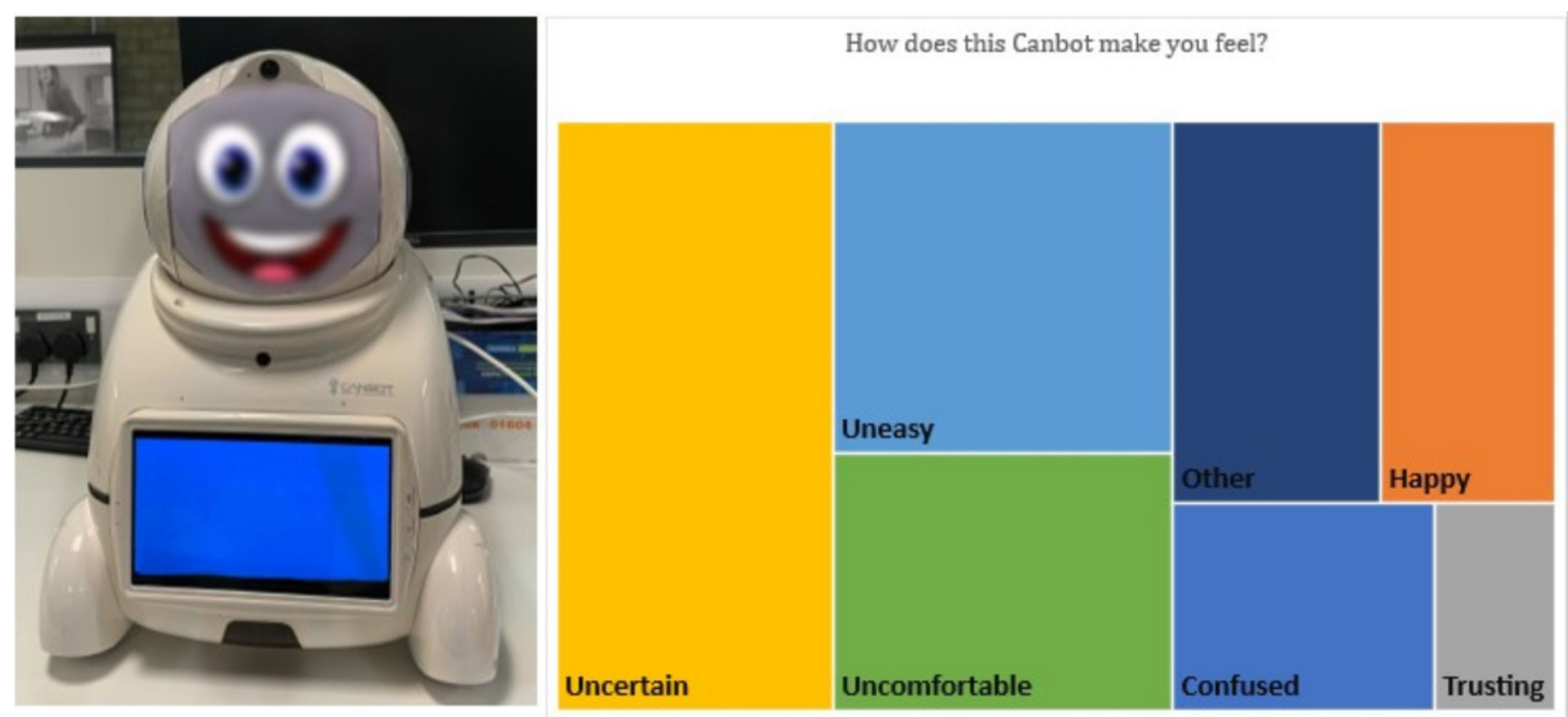


\section{Figure 6}

Heatmap displaying participants responses to: What robot would you trust is giving you the correct answer?
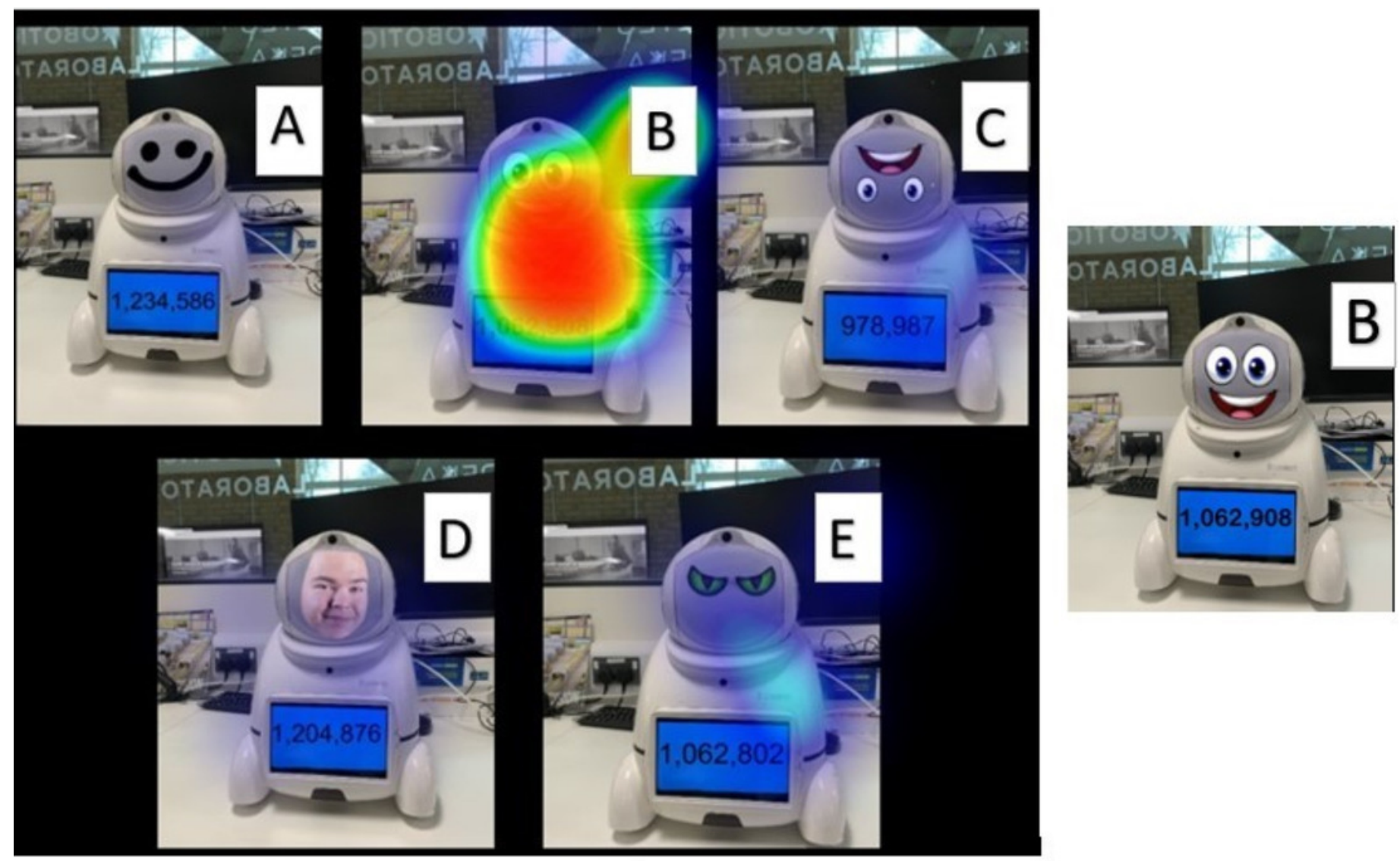


\section{Figure 7}

Heatmap displaying participants responses to: What robot would you trust is giving you the correct answer?

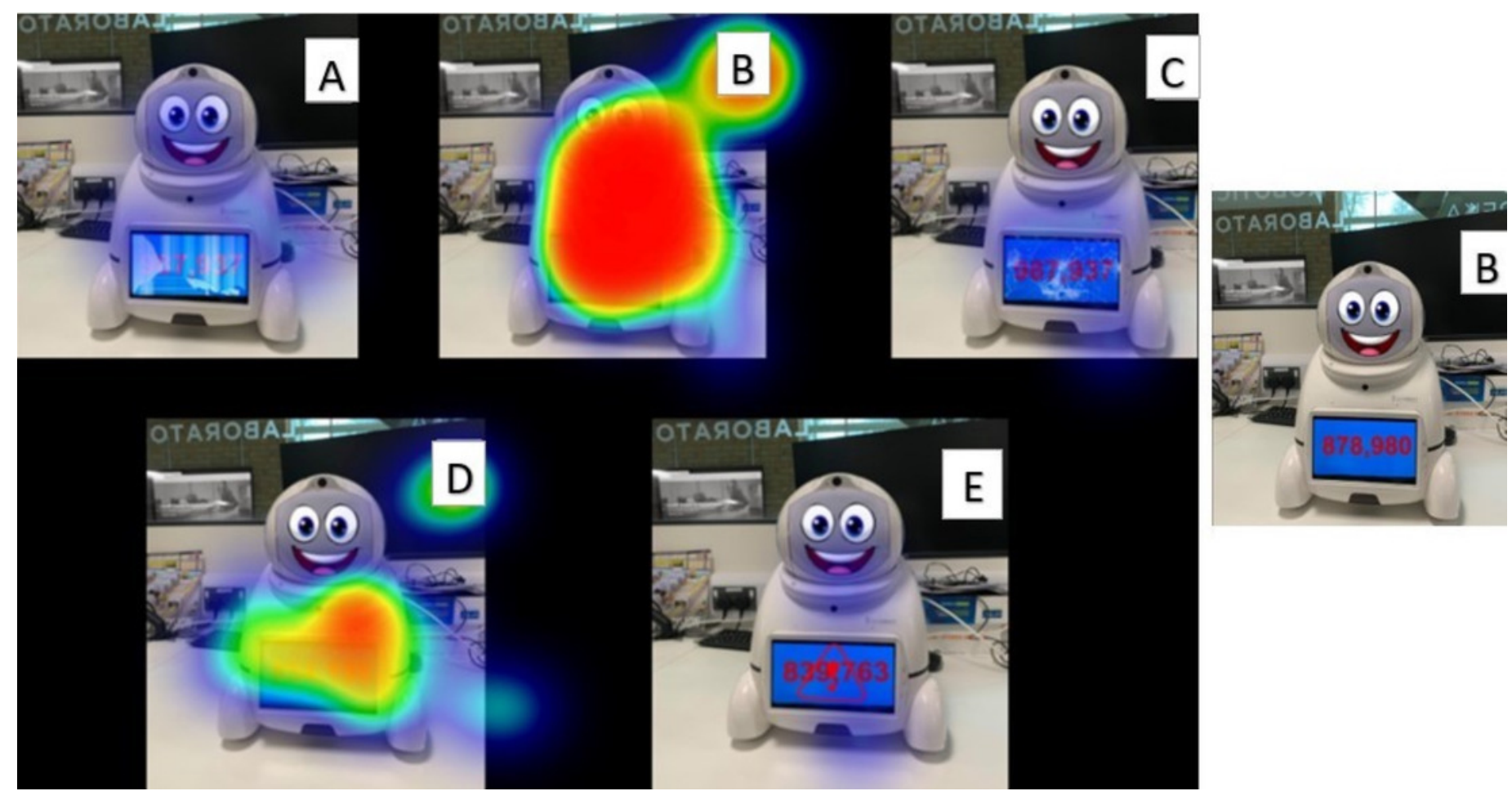




\section{Figure 8}

Robot with confused facial expression and participants responses to: Would you trust this robot is telling the truth about their age?

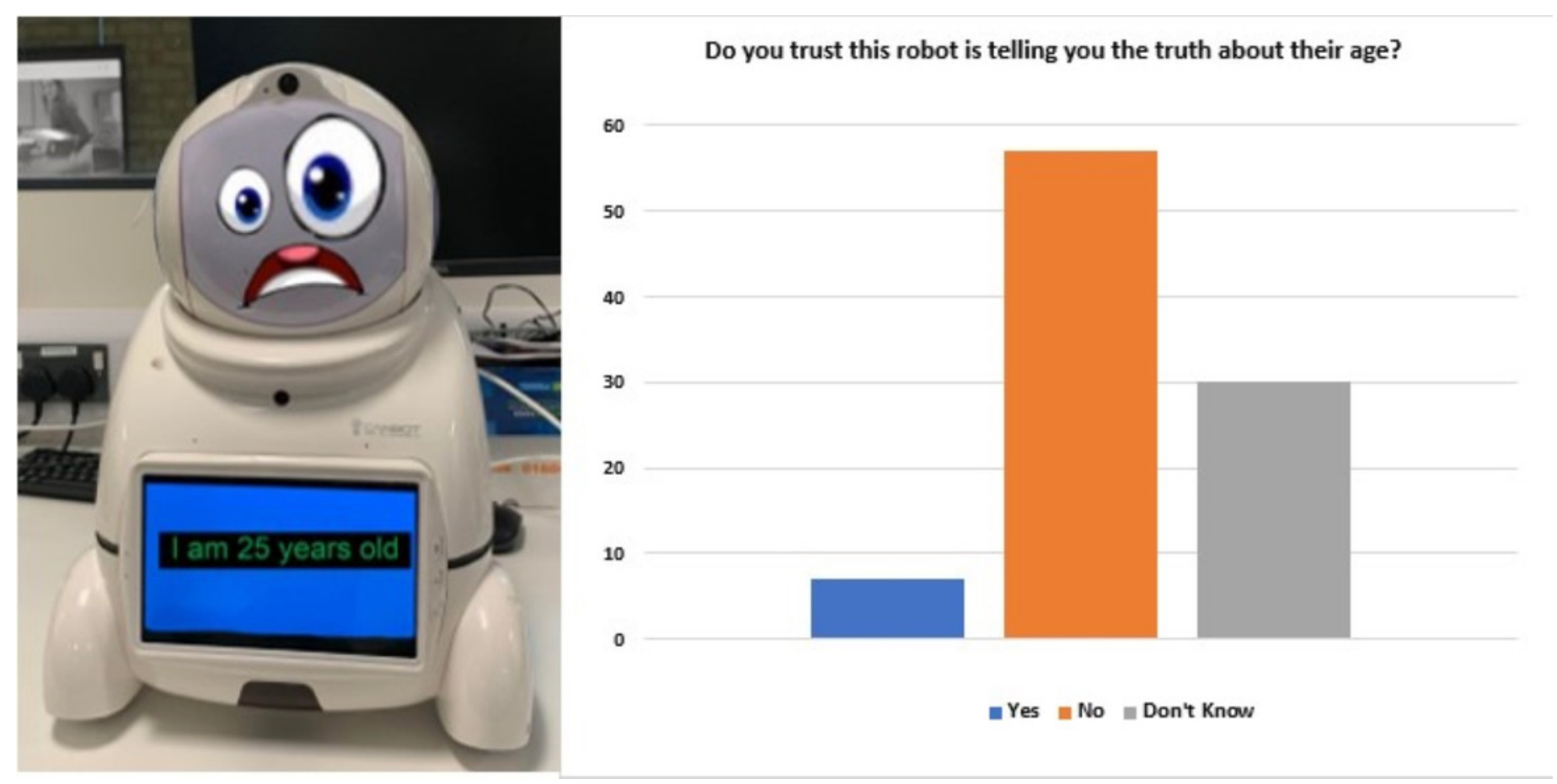




\section{Figure 9}

Sunburst visualisation displaying the visual modifications and participants associated wording.

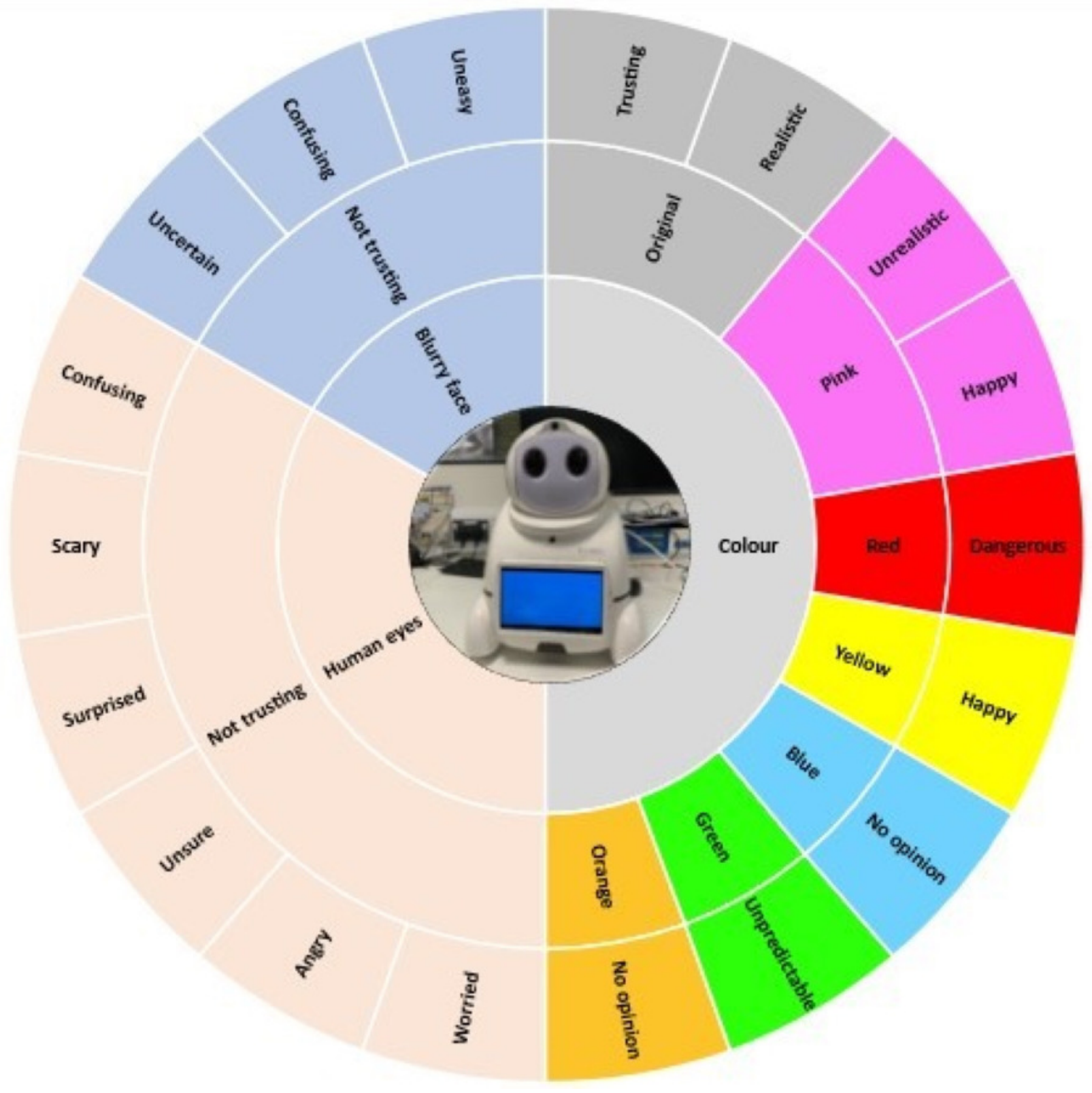

